COMMUNICATIONS IN

ANALYSIS AND GEOMETRY

Volume 11, Number 5, 1001-1026, 2003

\title{
Minimal Entropy Rigidity for Lattices in Products of Rank One Symmetric Spaces
}

\author{
Christopher Connell ${ }^{1}$ And Benson FArB $^{2}$
}

\section{Introduction.}

The volume entropy $h(g)$ of a closed Riemannian $n$-manifold $(M, g)$ is defined as

$$
h(g)=\lim _{R \rightarrow \infty} \frac{1}{R} \log (\operatorname{Vol}(B(x, R)))
$$

where $B(x, R)$ is the ball of radius $R$ around a fixed point $x$ in the universal cover $X$. (For noncompact $M$, see Section 6.2.) The number $h(g)$ is independent of the choice of $x$, and equals the topological entropy of the geodesic flow on $(M, g)$ when the curvature $K(g)$ satisfies $K(g) \leq 0$ (see [Ma]). Note that while the volume $\operatorname{Vol}(M, g)$ is not invariant under scaling the metric $g$, the normalized entropy

$$
\operatorname{ent}(g)=h(g)^{n} \operatorname{Vol}(M, g)
$$

is scale invariant.

Besson-Courtois-Gallot [BCG1] showed that, if $n \geq 3$ and $M$ admits a negatively curved, locally symmetric metric $g_{0}$, then ent $(g)$ is minimized uniquely by $g_{0}$ in the space of all Riemannian metrics on $M$. This striking result, called minimal entropy rigidity, has a great number of corollaries, including solutions to long-standing problems on geodesic flows, asymptotic harmonicity, Gromov's minvol invariant, and a new proof of Mostow Rigidity in the rank one case (see [BCG2]).

Extending minimal entropy rigidity to all nonpositively curved, locally symmetric manifolds $M$ has been a well-known open problem (see, e.g., [BCG2], Open Question 5). The case of closed manifolds locally (but not necessarily globally) isometric to products of negatively curved locally symmetric spaces of dimension at least 3 was announced in [BCG2] and later in [BCG3].

\footnotetext{
${ }^{1}$ Supported in part by an NSF postdoctoral fellowship.

${ }^{2}$ Supported in part by NSF grant DMS 9704640.
} 
In this paper we prove minimal entropy rigidity in this case as well as in the more general setting of complete, finite volume manifolds. Although we haven't seen Besson-Courtois-Gallot's proof of this result, it is likely that our proof (in the compact case) overlaps with theirs. In particular, we apply the powerful method introduced in [BCG1, BCG2], with a few new twists (see below).

Statement of result. While the quantity ent $(g)$ is invariant under scaling the metric on $M$, it is not invariant under scalings of the individual factors of $M$. Hence the iso-entropic inequality as in [BCG1, BCG2] does not hold as stated; one needs to find the locally symmetric metric $g_{\min }$ on $M$ which minimizes $h\left(g_{0}\right)^{n}$ for a fixed volume $\operatorname{Vol}\left(M, g_{0}\right)$ among all locally symmetric metrics $g_{0}$. This minimization problem is easily solved by Lagrange multipliers; as we show in $\S 2$, such a $g_{\text {min }}$ does indeed exist and is unique up to homothety.

We say that a Riemanninan manifold $X$ has bounded geometry if for any $\epsilon>0$ there exists $C$ so that every $\epsilon$-ball in $X$ has volume at least $C$. This holds for example when $X$ has injectivity radius bounded below and sectional curvature bounded above.

Theorem A (Minimal Entropy Rigidity). Let $M$ be an n-manifold which admits a complete, finite-volume Riemannian metric which is locally isometric to a product of negatively curved (rank 1) symmetric spaces of dimension at least 3 . Let $(N, g)$ be any finite volume Riemannian n-manifold such that $\widetilde{N}$ has bounded geometry. If $f: N \rightarrow M$ is any proper, coarsely Lipschitz map, then

$$
\operatorname{ent}(N, g) \geq|\operatorname{deg} f| \operatorname{ent}\left(M, g_{\min }\right)
$$

with equality iff $f$ is homotopic to a Riemannian covering, after possibly rescaling the metric on $M$ by a constant.

\section{Remarks.}

1. Taking $N=M$ in Theorem A gives the result that the locally symmetric $g_{\text {min }}$ minimizes entropy among all metrics on $M$, and in fact is the unique such metric up to homothety.

2. P. Verovic [Ve] has shown that Theorem A no longer holds if $g$ is allowed to be a Finsler metric, even when $f$ is the identity map. This behavior is different than in the rank one case. 
3. By similar arguments to those in [BCG1, BCG2], Theorem A implies strong (Mostow) rigidity for the corresponding locally symmetric manifolds.

\section{Outline of the proof of Theorem A.}

In this outline we assume $M$ and $N$ are compact. We will outline the proof of Remark 1 after Theorem A; this implies Theorem A by taking $f: N \rightarrow M$ to be the identity. For a moment consider the case when the metric on $N$ is nonpositively curved.

Endow $M$ with the unique locally symmetric metric $g_{\min }$ minimizing ent $(g)$. Denote by $Y$ (resp. $X$ ) the universal cover of $N$ (resp. $M$ ). Let $\mathcal{M}(\partial Y), \mathcal{M}(\partial X)$ denote the spaces of atomless probability measures on the visual boundaries $\partial Y, \partial X$ of the universal covers $Y, X$.

Morally what we do is, following the method of [BCG2], to define a map

$$
\tilde{F}: Y \rightarrow \mathcal{M}(\partial Y) \stackrel{\phi_{*}}{\rightarrow} \mathcal{M}(\partial X) \stackrel{\text { bar }}{\rightarrow} X
$$

where $\phi_{*}=\partial \tilde{f}_{*}$ is the pushforward of measures and bar is the "barycenter of a measure" (see $\S 4$ ). The inclusion $Y \rightarrow \mathcal{M}(\partial Y)$, denoted $x \mapsto \nu_{x}$, is given by the construction of the Patterson-Sullivan measures $\left\{\nu_{x}\right\}_{x \in X}$ corresponding to $\pi_{1}(N)<\operatorname{Isom}(Y)$ (see $\S 3$ ). An essential feature of these constructions is that they are all canonical, so that all of the maps are equivariant. Hence $\tilde{F}$ descends to a map $F: N \rightarrow M$.

One problem with this outline is that the metric on $Y$ is typically not nonpositively curved. In fact, if $Y$ is a finite volume nonpositively curved manifold diffeomorphic to an irreducible higher rank locally symmetric space then $Y$ is itself locally symmetric (see Chapter 9 of [Eb]). So we must find an alternative to using the "visual boundary" of $Y$. This is done by constructing smooth measures (parameterized by $s>h(g)$ ) on $Y$ itself, pushing them forward via $\tilde{f}$, and convolving with Patterson-Sullivan measure on $X$. The maps $\tilde{F}_{s}$ are then defined by taking the barycenters of these measures. In the equality case, the maps $F_{s}$ limit (as $s$ tends to $h(g)$ from above) to a Lipschitz map $F$ which turns out to be the desired locally isometric covering. This idea was first introduced in [BCG1].

As in [BCG1, BCG2], the main step in the proof is bounding the Jacobian $\left|\mathrm{Jac} F_{s}\right|$. A simple degree computation finishes the proof of the compact case. The extension to the noncompact, finite volume case is perhaps the most technical part of the proof, and requires extending some of the ideas of [BCS] to the higher rank setting to show that $F_{s}$ is proper. 
New features. As noted above, our proof is an application of the method of [BCG2]. The new features which occur in the present case are:

- In nonpositive curvature, Busemann functions are convex but not strictly convex; hence a global argument is needed to prove that the barycenter map is well-defined.

- As shown by Albuquerque [Al], each Patterson-Sullivan measure $\nu_{x}$ on a symmetric space $X$ is supported on a subset $\partial_{F} X \subset \partial X$ identified with the Furstenberg boundary. We make essential use of this fact in several places; in particular, the boundary $\partial_{F} X$ decomposes naturally as a product when $\operatorname{Isom}(X)$ does. The visual boundary $\partial X$ does not. The product decomposition of $\partial_{F} X$ allows one to reduce the key estimate on $\left|\operatorname{Jac} F_{s}\right|$ to the rank one case and an algebraic lemma of [BCG2]. Note that in the negatively curved case of [BCG1, BCG2], $\partial_{F} X=\partial X$.

- In the noncompact, finite volume case, direct geometric estimates on $N$ and $M$ and asymptotic analysis of the maps $F_{s}$ are used to show that the maps $F_{s}$ are proper.

\section{The best locally symmetric metric.}

In this section we find the locally symmetric metric $g_{\min }$ which minimizes $\operatorname{ent}\left(g_{0}\right)$ over all locally symmetric metrics $g_{0}$. Recall that the entropy $h\left(g_{0}\right)$ of a negatively curved, locally symmetric $n$-manifold $\left(M, g_{0}\right)$ with maximum sectional curvature -1 equals $n+d-2$, where $d=1,2$, 4 , or 8 according to whether $\left(M, g_{0}\right)$ is a real, complex, quaternionic, or Cayley hyperbolic space.

Now suppose that $\left(M, g_{0}\right)$ is a complete, finite volume Riemannian manifold which is locally isometric to a product

$$
\left(X_{1}, g_{1}\right) \times \cdots \times\left(X_{k}, g_{k}\right)
$$

of negatively curved symmetric spaces. After possibly scaling each factor, we may assume that $\left(X_{i}, g_{i}\right)$ has maximum sectional curvature -1 . In that case, $X_{i}$ has entropy $h_{i}=n_{i}+d_{i}-2$, where $n_{i}$ is the dimension of $X_{i}$ and $d_{i}=1,2,4$, or 8 is the dimension of the division algebra which classifies the symmetric space $X_{i}$. The space of locally symmetric metrics on $M$ is then the set of metrics

$$
g_{\beta}=\beta_{1}^{2} g_{1} \times \cdots \times \beta_{k}^{2} g_{k}
$$

where each $\beta_{i}$ is a positive real number. Note that the entropy of $\beta_{i}^{2} g_{i}$ is $\beta_{i}^{-1}\left(n_{i}+d_{i}-2\right)$. 
We now wish to minimize ent $\left(g_{\beta}\right)$ over all locally symmetric $g_{\beta}$ on $M$. This is the same as minimizing $h\left(g_{\beta}\right)$ while keeping volume fixed, i.e. while keeping $\prod_{i=1}^{k} \beta_{i}^{n_{i}}=1$.

It is not hard to see that the exponential growth rate of the volume of balls in the universal cover is given by $h_{0}=|l|$ where $l=\sum_{\alpha>0} m_{\alpha} \alpha^{*}$ where $\alpha^{*}$ is the dual of the positive root $\alpha$ with multiplicity $m_{\alpha}$ (see [Kn] for a sharp asympotic for the growth of balls). In the case of products of rank one spaces with entropies $\beta_{i}^{-1} h_{i}$, this becomes

$$
h\left(g_{\beta}\right)=\sqrt{\sum_{i=1}^{k} \beta_{i}^{-2} h_{i}^{2}}
$$

since the roots are all orthogonal with multiplicity $h_{i}$.

Now, with scalings as above, we wish to minimize

$$
h_{\beta}=\sqrt{\sum_{i=1}^{k} \beta_{i}^{-2} h_{i}^{2}}=\sqrt{\sum_{i=1}^{k} \beta_{i}^{-2}\left(n_{i}+d_{i}-2\right)^{2}}
$$

subject to $\prod_{i=1}^{k} \beta_{i}^{n_{i}}=1$. An easy computation using Lagrange multipliers now gives that the locally symmetric metric $g_{\min }$ which minimizes ent $(g)$ is

$$
g_{\min }=\alpha_{1}^{2} g_{1} \times \cdots \times \alpha_{k}^{2} g_{k}
$$

where

$$
\alpha_{i}=\frac{h_{i}}{\sqrt{n_{i}}} \prod_{i=1}^{k}\left(\frac{\sqrt{n_{i}}}{h_{i}}\right)^{\frac{n_{i}}{n}}
$$

In this case

$$
h\left(g_{\min }\right)=\sqrt{n} \prod_{i=1}^{k}\left(\frac{h_{i}}{\sqrt{n_{i}}}\right)^{n_{i} / n}
$$

\section{Patterson-Sullivan measures on symmetric spaces.}

In this section we briefly recall Albuquerque's theory [Al] of PattersonSullivan measures in higher rank symmetric spaces. For background on nonpositively curved manifolds, symmetric spaces, visual boundaries, Busemann functions, etc., we refer the reader to [BGS] and [Eb]. 


\subsection{Basic properties.}

Let $X$ be a Riemannian symmetric space of noncompact type. Denote by $\partial X$ the visual boundary of $X$; that is, the set of equivalence classes of geodesic rays in $X$, endowed with the cone topology. Hence $X \cup \partial X$ is a compactification of $X$ which is homeomorphic to a closed ball. Let $\Gamma$ be a lattice in $\operatorname{Isom}(X)$, so that $h\left(g_{0}\right)<\infty$ where $\left(M, g_{0}\right)$ is $\Gamma \backslash X$ with the induced metric.

Generalizing the construction of Patterson-Sullivan, Albuquerque constructs in $[\mathrm{Al}]$ a family of Patterson-Sullivan measures on $\partial X$. This is a family of probability measures $\left\{\nu_{x}\right\}_{x \in X}$ on $\partial X$ which provide a particularly natural embedding of $X$ into the space of measures on $\partial X$.

Proposition 3.1. The family $\left\{\nu_{x}\right\}$ of probability measures satisfies the following properties:

1. Each $\nu_{x}$ has no atoms.

2. The family of measures $\left\{\nu_{x}\right\}$ is $\operatorname{Isom}(X)$-equivariant:

$$
\gamma_{*} \nu_{x}=\nu_{\gamma x} \text { for all } \gamma \in \Gamma
$$

3. For all $x, y \in X$, the measure $\nu_{y}$ is absolutely continuous with respect to $\nu_{x}$. In fact the Radon-Nikodym derivative is given explicitly by:

$$
\frac{d \nu_{x}}{d \nu_{y}}(\xi)=e^{h(g) B(x, y, \xi)}
$$

where $B(x, y, \xi)$ is the Busemann function on $X$. For points $x, y \in X$ and $\xi \in \partial X$, the function $B: X \times X \times \partial X \rightarrow \mathbb{R}$ is defined by

$$
B(x, y, \xi)=\lim _{t \rightarrow \infty} d_{X}\left(y, \gamma_{\xi}(t)\right)-t
$$

where $\gamma_{\xi}$ is the unique geodesic ray with $\gamma(0)=x$ and $\gamma(\infty)=\xi$.

Since Isom $(X)$ acts transitively on $X$, the first property above implies that the $\nu_{x}$ are all probability measures. The second property implies no two measures are the same. Thus the assignment $x \mapsto \nu_{x}$ defines an injective map

$$
\nu: X \rightarrow \mathcal{M}(\partial X)
$$

where $\mathcal{M}(\partial X)$ is the space of probability measures on $X$. Such a mapping satisfying the above properties is called an $h\left(g_{0}\right)$-conformal density. 


\subsection{The Furstenberg boundary and Albuquerque's Theorem.}

The Furstenberg boundary of a symmetric space $X$ of noncompact type is abstractly defined to be $G / P$ where $P$ is a minimal parabolic subgroup of the connected component $G$ of the identity of $\operatorname{Isom}(X)$.

Fix once and for all a basepoint $p \in X$. This choice uniquely determines a Cartan decomposition $\mathfrak{g}=\mathfrak{k} \oplus \mathfrak{p}$ of the Lie algebra of $G$ where $\mathfrak{k}$ is the Lie algebra of the isotropy subgroup $K=\operatorname{Stab}_{G}(p)$ of $p$ in $G$ and $\mathfrak{p}$ is identified with the tangent space $T_{p} X$.

Let $\mathfrak{a}$ be a fixed maximal abelian subspace of $\mathfrak{p}$. The rank of $X$, denoted $\operatorname{rank}(X)$, is the dimension of $\mathfrak{a}$. If $A=\exp (\mathfrak{a})$ then $A \cdot p$ will be a maximal flat (totally geodesically embedded Euclidean space of maximal dimension). Recall, a vector $v \in T X$ is called a regular vector if it is tangent to a unique maximal flat. Otherwise it is a singular vector. A geodesic is called regular (resp. singular) if one (and hence all) of its tangent vectors are regular (singular). A point $\xi \in \partial X$ is regular (singular) if any (and hence all) of the geodesics in the corresponding equivalence class are regular (singular).

A Weyl chamber $\mathfrak{a}^{+}$is a choice of connected component of the set of regular vectors in $\mathfrak{a}$. There corresponds to $\mathfrak{a}^{+}$a choice of positive roots. Similarly if $A^{+}=\exp \left(\mathfrak{a}^{+}\right)$then $A^{+} \cdot p$ is called a Weyl chamber of the flat $A \cdot p$. The union of all the singular geodesics in the flat $A \cdot p$ passing through $p$ is a finite set of hyperplanes forming the boundaries of the Weyl chambers.

In our current special case of products, $\operatorname{rank}(X)=k$ (recall $k$ is the number of factors) since a product of geodesics, one from each factor, produces a maximal flat. Also, the Weyl chambers are simply the $2^{\operatorname{rank}(X)}$ orthants of $\mathfrak{a} \simeq \mathbb{R}^{\operatorname{rank}(X)}$ and $b^{+}$is the unit vector corresponding to the barycenter of the extremal unit vectors on the interior boundary of a fixed Weyl chamber.

The Furstenberg boundary can be identified with the orbit of $G$ acting on any regular point $v(\infty) \in \partial X$, the endpoint of a geodesic tangent to a regular vector $v$. of a Weyl chamber in a fixed flat $\mathfrak{a}$. This follows from the fact that the action of any such $P$ on $\partial X$ fixes some regular point.

Because of this, for symmetric spaces of higher rank, behaviour on the visual boundary can often be aptly described by its restriction to the Furstenberg boundary. Here we will use only some very basic properties of this boundary. For more details on semisimple Lie groups and the Furstenberg boundary, see $[\mathrm{Zi}]$.

Let $b$ be the sum of the dual vectors of the positive roots corresponding to $\mathfrak{a}^{+}$. The vector $b^{+}$is called the algebraic centroid of $\mathfrak{a}^{+}$. Set $b^{+}=b /\|b\|$.

Define the set $\partial_{F} X \subset \partial X$ to be $\partial_{F} X=G \cdot b^{+}(\infty)$. Henceforth we will refer to the Furstenberg boundary as this specific realization. We point out 
that for any lattice $\Gamma$ in $\operatorname{Isom}(X)$, the induced action on the boundary is transitive only on $\partial_{F} X$. That is, $\overline{\Gamma \cdot b^{+}(\infty)}=G \cdot b^{+}(\infty)$ even though for any interior point $x \in X, \overline{\Gamma \cdot x}=\partial X$.

Theorem 7.4 and Proposition 7.5 of $[\mathrm{Al}]$ combine to give the following categorization which will play a crucial role in our proof of Theorem A.

Theorem 3.2 (Description of $\left.\nu_{x}\right)$. Let $\left(X, g_{0}\right)$ be a symmetric space of noncompact type, and let $\Gamma$ be a lattice in $\operatorname{Isom}(X)$. Then

1. $h\left(g_{0}\right)=\|b\|$,

2. $b^{+}(\infty)$ is a regular point, and hence $\partial_{F} X$ is a regular set,

3. For any $x \in X$, the support $\operatorname{supp}\left(\nu_{x}\right)$ of $\nu_{x}$ is equal to $\partial_{F} X$, and

4. $\nu_{x}$ is the unique probability measure invariant under the action on $\partial_{F} X$ of the compact isotropy group $\operatorname{Stab}_{G}(x)$ at $x$. In particular, $\nu_{p}$ is the unique $K$-invariant probability measure on $\partial_{F} X$.

Note that when $X$ is has rank one, $\partial_{F} X=\partial X$. In general $\partial_{F} X$ has codimension $\operatorname{rank}(X)-1$ in $\partial X$.

\subsection{Limits of Patterson-Sullivan measures.}

We now describe the asymptotic behaviour of the $\nu_{x}$ as $x$ tends to a point in $\partial X$.

For any point $\xi$ of the visual boundary, let $S_{\theta}$ be the set of points $\xi \in \partial_{F} X$ such that there is a Weyl chamber $W$ whose closure $\partial \bar{W}$ in $\partial X$ contains both $\theta$ and $\xi$. Let $K_{\theta}$ be the subgroup of $K$ which stabilizes $S_{\theta} . K_{\theta}$ acts transitively on $S_{\theta}$ (see the proof below).

Theorem 3.3 (Support of $\nu_{x}$ ). Given any sequence $\left\{x_{i}\right\}$ tending to $\theta \in$ $\partial X$ in the cone topology, the measures $\nu_{x_{i}}$ converge in $\mathcal{M}\left(\partial_{F} X\right)$ to the unique $K_{\theta}$-invariant probability measure $\nu_{\theta}$ supported on $S_{\theta}$.

Proof. Let $x_{i}=g_{i} \cdot p$, for an appropriate sequence $g_{i} \in G$. Recall that $\nu_{x_{i}}=\left(g_{i}\right)_{*} \nu_{p}$. Then combining part (4) of Theorem 3.2 with Proposition 9.43 of [GJT] have that some subsequence of the $\nu_{x_{i}}$ converges to a $K_{\theta^{-}}$ invariant measure $\nu_{\theta}$ supported on $S_{\theta}$.

Note that in [GJT], the notation $I$ refers to a subset of a fundamental set of roots corresponding to the face of a Weyl chamber containing $\theta$ in its 
boundary. If $g_{i} \cdot p=k_{i} a_{i} \cdot p$ converges then both $k=\lim k_{i}$ and $a^{I}=\lim _{i} a_{i}^{I}$ exist (note the definition of $a^{I}$ in [GJT]). Again in the notation of [GJT], $K_{\theta}$ is the conjugate subgroup $\left(k a^{I}\right) K^{I}\left(k a^{I}\right)^{-1}$ in $K$. Moreover, $S_{\theta}$ is the orbit $k a^{I} K^{I} \cdot b^{+}(\infty)$.

By Corollary 9.46 and Proposition 9.45 of [GJT], any other convergent subsequence of the $\nu_{x_{i}}$ produces the same measure in the limit, and therefore the sequence $\nu_{x_{i}}$ itself converges to $\nu_{\theta}$ uniquely.

In the case when $\theta$ is a regular point, the above theorem implies that $S_{\theta}$ is a single point and the limit measure $\nu_{\theta}$ is simply the Dirac probability measure at that point point in $\partial_{F} X$.

\section{The barycenter of a measure.}

In this section we describe the natural map which is an essential ingredient in the method of Besson-Courtois-Gallot.

Let $\phi$ denote the lift to universal covers of $f$ with basepoint $p \in Y$ (resp. $f(p) \in X$ ), i.e. $\phi=\tilde{f}: Y \rightarrow X$. We will also denote the metric and Riemannian volume form on universal cover $Y$ by $g$ and $d g$ respectively. Then for each $s>h(g)$ and $y \in Y$ consider the probability measure $\mu_{y}^{s}$ on $Y$ in the Lebesgue class with density given by

$$
\frac{d \mu_{y}^{s}}{d g}(z)=\frac{e^{-s d(y, z)}}{\int_{Y} e^{-s d(y, z)} d g}
$$

The $\mu_{y}^{s}$ are well defined by the choice of $s$.

Consider the push-forward $\phi_{*} \mu_{y}^{s}$, which is a measure on $X$. Define $\sigma_{y}^{s}$ to be the convolution of $\phi_{*} \mu_{y}^{s}$ with the Patterson-Sullivan measure $\nu_{z}$ for the symmetric metric.

More precisely, for $U \subset \partial X$ a Borel set, define

$$
\sigma_{y}^{s}(U)=\int_{X} \nu_{z}(U) d\left(\phi_{*} \mu_{y}^{s}\right)(z)
$$

Since $\left\|\nu_{z}\right\|=1$, we have

$$
\left\|\sigma_{y}^{s}\right\|=\left\|\mu_{y}^{s}\right\|=1
$$

Let $B_{0}(x, \theta)=B_{0}(\phi(p), x, \theta)$ be the Busemann function on $X$ with respect to the basepoint $\phi(p)$ (which we will also denote by $p$ ). For $s>h(g)$ and $x \in X, y \in Y$ define a function

$$
\mathcal{B}_{s, y}(x)=\int_{\partial X} B_{0}(x, \theta) d \sigma_{y}^{s}(\theta)
$$


By Theorem 3.3, the support of $\nu_{z}$, hence of $\sigma_{y}^{s}$, is all of $\partial_{F} X$, which in turn equals the $G$-orbit $G \cdot b^{+}(\infty)$, where $G=\operatorname{Isom}\left(X_{0}\right)$. Hence

$$
\mathcal{B}_{s, y}(x)=\int_{\partial_{F} X} B_{0}(x, \theta) d \sigma_{y}^{s}(\theta)=\int_{G \cdot \xi} B_{0}(x, \theta) d \sigma_{y}^{s}(\theta)
$$

Since $X$ is nonpositively curved, the Busemann function $B_{0}$ is convex on $X$. Hence $\mathcal{B}_{s, y}$ is convex on $X$, being a convex integral of convex functions. While $B_{0}$ is strictly convex only when $X$ is negatively curved, we have the following.

Proposition 4.1 (Strict convexity of $\mathcal{B}$ ). For each fixed $y$ and $s$, the function $x \mapsto \mathcal{B}_{y, s}(x)$ is strictly convex, and has a unique critical point in $X$ which is its minimum.

Proof. For the strict convexity, it suffices to show that given a geodesic segment $\gamma(t)$ between two points $\gamma(0), \gamma(1) \in X$, there exists some $\xi \in \partial_{F} X$ such that function $B_{0}(\gamma(t), \xi)$ is strictly convex in $t$, and hence on an open positive $\sigma_{y}^{s}$-measure set around $\xi$. We know it is convex by the comment preceding the statement of the proposition.

If $B_{0}(\gamma(t), \xi)$ is constant on some geodesic subsegment of $\gamma$ for some $\xi$, then $\gamma$ must lie in some flat $\mathcal{F}$ such that the geodesic between $\xi \in \partial \mathcal{F}$ and $\gamma$ (which meets $\gamma$ at a right angle) also lies in $\mathcal{F}$. On the other hand, $\xi \in \partial_{F} X$ is in the direction of the algebraic centroid in a Weyl chamber, and $\gamma$ is perpendicular to this direction. By the properties of the roots, $\gamma$ is a regular geodesic (not contained in the boundary of a Weyl chamber). In particular, $\gamma$ is contained in exactly one flat $\mathcal{F}$. Furthermore, $\partial_{F} X \cap \partial \mathcal{F}$ is a finite set (an orbit of the Weyl group). As a result, for almost every $\xi \in \partial_{F} X$ $B_{0}(\gamma(t), \xi)$ is strictly convex in $t$.

For fixed $z \in X$, by the last property listed in Proposition 3.1, we see that

$$
\int_{\partial_{F} X} B_{0}(x, \theta) d \nu_{z}(\theta)
$$

tends to $\infty$ as $x$ tends to any boundary point $\xi \in \partial X$. Then for fixed $y$ and $s>h(g), \mathcal{B}_{y, s}(x)$ increases to $\infty$ as $x$ tends to any boundary point $\xi \in \partial X$. Hence it has a local minimum in $X$, which by strict convexity must be unique.

We call the unique critical point of $\mathcal{B}_{s, y}$ the barycenter of the measure 
$\sigma_{y}^{s}$, and define a map $\tilde{F}_{s}: Y \rightarrow X$ by

$$
\tilde{F}_{s}(y)=\text { the unique critical point of } \mathcal{B}_{s, y}
$$

Since for any two points $p_{1}, p_{2} \in X$ we have that

$$
B_{0}\left(p_{1}, x, \theta\right)=B_{0}\left(p_{2}, x, \theta\right)+B_{0}\left(p_{1}, p_{2}, \theta\right)
$$

it follows that $\mathcal{B}_{s, y}$ only changes by an additive constant when we change the basepoint of $B_{0}$. This change does not affect the location of critical point of $\mathcal{B}_{y, s}$. As a result, $\tilde{F}_{s}$ is independent of choice of basepoints.

The equivariance of $\phi$ and of $\left\{\mu_{y}\right\}$ implies that $\tilde{F}_{s}$ is also equivariant. Hence $\tilde{F}_{s}$ descends to a map $F_{s}: N \rightarrow M$. As in [BCG1], we will see that $F_{s}$ is $C^{1}$, and will estimate its Jacobian.

\section{The Jacobian estimate.}

The goal of this section is to prove a sharp estimate on the magnitude of the Jacobian of $F_{s}: N \rightarrow M$.

We obtain the differential of $F_{s}$ by implicit differentiation:

$$
0=D_{x=F_{s}(y)} \mathcal{B}_{s, y}(x)=\int_{\partial_{F} X} d B_{0\left(F_{s}(y), \theta\right)}(\cdot) d \sigma_{y}^{s}(\theta)
$$

Hence as two forms we have:

$$
\begin{aligned}
0= & D_{y} D_{x=F_{s}(y)} \mathcal{B}_{s, y}(x)=\int_{\partial_{F} X} D d B_{0\left(F_{s}(y), \theta\right)}\left(D_{y} F_{s}(\cdot), \cdot\right) d \sigma_{y}^{s}(\theta) \\
& -s \int_{Y} \int_{\partial_{F} X} d B_{0\left(F_{s}(y), \theta\right)}(\cdot)\left\langle\nabla_{y} d_{Y}(y, z), \cdot\right\rangle d \nu_{\phi(z)}(\theta) d \mu_{y}^{s}(z)
\end{aligned}
$$

The distance function $d_{Y}(y, z)$ is Lipschitz and $C^{1}$ off of the cut locus which has Lebesgue measure 0. It follows from the Implicit Function Theorem (see [BCG2]) that $F_{s}$ is $C^{1}$ for $s>h(g)$. By chain rule,

$$
\operatorname{Jac} F_{s}=s^{n} \frac{\operatorname{det}\left(\int_{Y} \int_{\partial_{F} X} d B_{0\left(F_{s}(y), \theta\right)}(\cdot)\left\langle\nabla_{y} d(y, z), \cdot\right\rangle d \nu_{\phi(z)}(\theta) d \mu_{y}^{s}(z)\right)}{\operatorname{det}\left(\int_{\partial_{F} X} D d B_{0\left(F_{s}(y), \theta\right)}(\cdot, \cdot) d \sigma_{y}^{s}(\theta)\right)}
$$

Applying Hölder's inequality to the numerator gives:

$\left|\operatorname{Jac} F_{s}\right| \leq s^{n} \frac{\operatorname{det}\left(\int_{\partial_{F} X} d B_{0}^{2}{ }_{\left(F_{s}(y), \theta\right)} d \sigma_{y}^{s}(\theta)\right)^{1 / 2} \operatorname{det}\left(\int_{Y}\left\langle\nabla_{y} d_{Y}(y, z), \cdot\right\rangle^{2} d \mu_{y}^{s}(z)\right)^{1 / 2}}{\operatorname{det}\left(\int_{\partial_{F} X} D d B_{0\left(F_{s}(y), \theta\right)}\left(D_{y} F_{s}(\cdot), \cdot\right) d \sigma_{y}^{s}(\theta)\right)}$ 
Using that $\operatorname{Tr}\left\langle\nabla_{y} d_{Y}(y, z), \cdot\right\rangle^{2}=\left|\nabla_{y} d_{Y}(y, z)\right|^{2}=1$, except possibly on a measure 0 set, we may estimate

$$
\operatorname{det}\left(\int_{Y}\left\langle\nabla_{y} d_{Y}(y, z), \cdot\right\rangle^{2} d \mu_{y}^{s}(z)\right)^{1 / 2} \leq\left(\frac{1}{\sqrt{n}}\right)^{n}
$$

Therefore

$$
\left|\operatorname{Jac} F_{s}\right| \leq\left(\frac{s}{\sqrt{n}}\right)^{n} \frac{\operatorname{det}\left(\int_{\partial_{F} X} d B_{0}^{2}{ }_{\left(F_{s}(y), \theta\right)} d \sigma_{y}^{s}(\theta)\right)^{1 / 2}}{\operatorname{det}\left(\int_{\partial_{F} X} D d B_{0\left(F_{s}(y), \theta\right)}\left(D_{y} F_{s}(\cdot), \cdot\right) d \sigma_{y}^{s}(\theta)\right)} .
$$

Theorem 5.1 (The Jacobian Estimate). For all $s>h(g)$ and all $y \in N$ we have:

$$
\left|\operatorname{Jac} F_{s}(y)\right| \leqslant\left(\frac{s}{h\left(g_{\min }\right)}\right)^{n}
$$

with equality at any $y \in N$ if and only if $D_{y} F_{s}$ is a homothety.

As discussed in the introduction, the main idea is to use the fact that the measures $\sigma_{y}^{s}$ on $\partial X$ are supported on $\partial_{F} X$, which decomposes as a product of Furtsenberg boundaries of the rank one factors of $X$. This can then be used to reduce the estimate on $\left|\operatorname{Jac} F_{s}(y)\right|$ to the rank one case and an algebraic lemma of [BCG1].

Proof. Item (1) is clear; we prove item (2).

By the hypothesis on $X$, the group $G=\operatorname{Isom}(X)$ can be written as a product $G=G_{1} \times G_{2} \cdots \times G_{\operatorname{rank}(X)}$, where each $G_{i} \neq \mathrm{SL}(2, \mathbb{R})$ is a simple rank one Lie group. Theorem 3.3 states that there exists $\xi \in \partial X$ so that for all $y \in Y$, the measure $\sigma_{y}^{s}$ is supported on some $G$-orbit

$$
G \cdot \xi=\left\{\left(G_{1} \times G_{2} \cdots \times G_{\operatorname{rank}(X)}\right) \cdot \xi\right\}
$$

Hence

$$
\partial_{F} X=G \cdot \xi=\partial_{F} X_{1} \times \cdot \times \partial_{F} X_{\operatorname{rank}(X)}
$$

Since each $X_{i}$ has rank one, $\partial_{F} X_{i}=\partial X_{i}$ so that

$$
\partial_{F} X=\partial X_{1} \times \cdots \times \partial X_{\operatorname{rank}(X)}
$$

Let $B_{i}$ denote the Busemann function for the rank one symmetric space $X_{i}$ with metric $g_{i}$. Then for $\theta_{i} \in \partial X_{i} \subset \partial X$ and $x, y \in X_{i}$ we have $B_{0}\left(x, y, \theta_{i}\right)=\alpha_{i} B_{i}\left(x, y, \theta_{i}\right)$. Since the factors $X_{i}$ are orthogonal in $X$ with 
respect to the metric $g_{\min }$, the Busemann function of $\left(X, g_{\min }\right)$ with basepoint $p \in X$ at a point $\theta=\left(\theta_{1}, \ldots, \theta_{\operatorname{rank}(X)}\right) \in \partial_{F} X$ is given by

$$
B_{0}(x, \theta)=\sum_{i=1}^{\operatorname{rank}(X)} \frac{\alpha_{i}}{\sqrt{\operatorname{rank}(X)}} B_{i}\left(x_{i}, \theta_{i}\right)
$$

Since $\nabla_{x}^{g_{\min }} B_{i}=\frac{1}{\alpha_{i}^{2}} \nabla_{x}^{g_{i}} B_{i}$, we may verify that $\left|\nabla_{x}^{g_{\min }} B_{0}(x, \theta)\right|_{g_{\min }}^{2}=1$. Similarly,

$$
\nabla_{x}^{g_{\min }} B_{0}=\sum_{i=1}^{\operatorname{rank}(X)} \frac{1}{\alpha_{i} \sqrt{\operatorname{rank}(X)}} \nabla_{x}^{g_{i}} B_{i}
$$

Differentiating again with respect to a fixed orthonormal basis of $T_{x} X$ with respect to $g_{\min }$ we obtain,

$$
\operatorname{Hess}_{x}^{g_{\min }} B_{0}=\oplus_{i=1}^{\operatorname{rank}(X)} \frac{1}{\alpha_{i} \sqrt{\operatorname{rank}(X)}} \operatorname{Hess}_{x}^{g_{i}} B_{i}
$$

Since $\sigma_{y}^{s}$ is supported on $G_{1} \times \cdots \times G_{\operatorname{rank}(X)} \cdot \xi$, we can use product coordinates on $X$ to write the right hand side of (4) as,

$$
\left(\frac{s}{\sqrt{n}}\right)^{n} \frac{\operatorname{det}\left(\int_{\partial_{F} X}\left(\sum_{i=1}^{\operatorname{rank}(X)} \nabla_{\left(F_{s}(y), \theta_{i}\right)}^{g_{i}} B_{i}\right)\left(\sum_{i=1}^{\operatorname{rank}(X)} \nabla_{\left(F_{s}(y), \theta_{i}\right)}^{g_{i}} B_{i}\right)^{*} d \sigma_{y}^{s}(\theta)\right)^{\frac{1}{2}}}{\operatorname{det}\left(\int_{\partial_{F} X} \oplus_{i=1}^{\operatorname{rank}(X)} \operatorname{Hess}_{\left(F_{s}(y), \theta_{i}\right)}^{g_{i}} B_{i} d \sigma_{y}^{s}(\theta)\right)}
$$

where the superscript $*$ means transpose and we have cancelled the factor $\left(\frac{1}{\alpha_{i} \sqrt{\operatorname{rank}(X)}}\right)^{n}$ from the numerator and denominator.

Recall the following estimate for positive semi-definite block matrices,

$$
\operatorname{det}\left(\begin{array}{cc}
A & B \\
B^{*} & C
\end{array}\right) \leqslant \operatorname{det}(A) \operatorname{det}(C)
$$

Then since the matrix

$$
\int_{\partial_{F} X}\left(\sum_{i=1}^{\operatorname{rank}(X)} \nabla_{\left(F_{s}(y), \theta_{i}\right)}^{g_{i}} B_{i}\right)\left(\sum_{i=1}^{\operatorname{rank}(X)} \nabla_{\left(F_{s}(y), \theta_{i}\right)}^{g_{i}} B_{i}\right)^{*} d \sigma_{y}^{s}(\theta)
$$

is positive semi-definite, by iteratively using the above estimate (on sub- 
blocks) we obtain

$$
\begin{array}{r}
\operatorname{det}\left(\int_{\partial_{F} X}\left(\sum_{i=1}^{\operatorname{rank}(X)} \nabla_{\left(F_{s}(y), \theta_{i}\right)}^{g_{i}} B_{i}\right)\left(\sum_{i=1}^{\operatorname{rank}(X)} \nabla_{\left(F_{s}(y), \theta_{i}\right)}^{g_{i}} B_{i}\right)^{*} d \sigma_{y}^{s}(\theta)\right) \leq \\
\prod_{i=1}^{\operatorname{rank}(X)} \operatorname{det}\left(\int_{\partial_{F} X}\left(\nabla_{\left(F_{s}(y), \theta_{i}\right)}^{g_{i}} B_{i}\right)\left(\nabla_{\left(F_{s}(y), \theta_{i}\right)}^{g_{i}} B_{i}\right)^{*} d \sigma_{y}^{s}(\theta)\right)
\end{array}
$$

Also, we have

$$
\operatorname{det}\left(\int_{\partial_{F} X} \oplus_{i=1}^{\operatorname{rank}(X)} \operatorname{Hess}_{\left(F_{s}(y), \theta_{i}\right)}^{g_{i}} B_{i} d \sigma_{y}^{s}(\theta)\right)=\prod_{i=1}^{\operatorname{rank}(X)} \operatorname{det}\left(\int_{\partial_{F} X} \operatorname{Hess}_{\left(F_{s}(y), \theta_{i}\right)}^{g_{i}} B_{i} d \sigma_{y}^{s}(\theta)\right)
$$

Hence we have

$\left|\operatorname{Jac} F_{s}(y)\right| \leq\left(\frac{s}{\sqrt{n}}\right)^{n} \prod_{i=1}^{\operatorname{rank}(X)} \frac{\operatorname{det}\left(\int_{\partial_{F} X}\left(\nabla_{\left(F_{s}(y), \theta_{i}\right)}^{g_{i}} B_{i}\right)\left(\nabla_{\left(F_{s}(y), \theta_{i}\right)}^{g_{i}} B_{i}\right)^{*} d \sigma_{y}^{s}(\theta)\right)^{1 / 2}}{\operatorname{det}\left(\int_{\partial_{F} X} \operatorname{Hess}_{\left(F_{s}(y), \theta_{i}\right)}^{g_{i}} B_{i} d \sigma_{y}^{s}(\theta)\right)}$

Since $B_{i}$ is the Busemann function of rank one symmetric space $X_{i}$, it follows that, setting the tensor $H_{i}=\left(\nabla_{\left(F_{s}(y), \theta\right)}^{g_{i}} B_{i}\right)^{2}$, we have

$$
\operatorname{Hess}_{\left(F_{s}(y), \theta_{i}\right)}^{g_{i}} B_{i}=\mathrm{I}-H_{i}-\sum_{k=1}^{d_{i}-1} J_{k} H_{i} J_{k}
$$

where the $J_{k}$ are the matrices representing the underlying complex structure of (the division algebra corresponding to) the symmetric space. Lemma 5.5 of [BCG2] says that for any $n \times n$ matrix $H$ with $\operatorname{Tr} H=1$, the following holds:

$$
\frac{\operatorname{det} H^{1 / 2}}{\operatorname{det}\left(\mathrm{I}-H-\sum_{k=1}^{d_{i}-1} J_{k} H J_{k}\right)} \leq\left(\frac{\sqrt{n}}{n+d-2}\right)^{n}
$$

with equality if and only if $H=\frac{1}{n} \mathrm{I}$. Applying this estimate to each term of the above product now gives

$$
\frac{\operatorname{det}\left(\int_{\partial_{F} X}\left(\nabla_{\left(F_{s}(y), \theta_{i}\right)}^{g_{i}} B_{i}\right)\left(\nabla_{\left(F_{s}(y), \theta_{i}\right)}^{g_{i}} B_{i}\right)^{*} d \sigma_{y}^{s}(\theta)\right)^{1 / 2}}{\operatorname{det}\left(\int_{\partial_{F} X} \operatorname{Hess}_{\left(F_{s}(y), \theta_{i}\right)}^{g_{i}} B_{i} d \sigma_{y}^{s}(\theta)\right)} \leq\left(\frac{\sqrt{n_{i}}}{n_{i}+d_{i}-2}\right)^{n_{i}}
$$


with equality if and only if $H_{i}=\frac{1}{n_{i}} \mathrm{I}$. Hence

$$
\left|\operatorname{Jac} F_{s}(y)\right| \leq\left(\frac{s}{\sqrt{n}}\right)^{n} \prod_{i=1}^{\operatorname{rank}(X)}\left(\frac{\sqrt{n_{i}}}{n_{i}+d_{i}-2}\right)^{n_{i}}=\frac{s^{n}}{h\left(g_{\min }\right)^{n}}
$$

If equality is attained then we have equality for each term. Let $Q_{i} \subset$ $T_{y} Y$ be the subspace which is mapped by $D_{y} F_{s}$ onto $T_{F_{s}(y)} X_{i}$. So for any $v_{i} \in T_{F_{s}(y)} X_{i}$ and any $u_{i} \in Q_{i}$ we have

$$
\left|\left\langle D_{y} F_{s}\left(u_{i}\right), v_{i}\right\rangle_{g_{i}}\right| \leq \frac{\sqrt{n} \sqrt{n_{i}} s\left\|v_{i}\right\|_{g_{i}}}{n_{i}+d_{i}-2}\left(\int_{\partial_{F} X} d B_{y, \phi^{-1}(\theta)}\left(u_{i}\right)^{2} d \sigma_{y}^{s}(\theta)\right)^{1 / 2}
$$

Recall that $\alpha_{i}=\frac{h_{i}}{\sqrt{n_{i}}} \prod_{i=1}^{\operatorname{rank}(X)}\left(\frac{\sqrt{n_{i}}}{h_{i}}\right)^{\frac{n_{i}}{n}}$. So multiplying each side by $\alpha_{i}^{2}$ we write the above with respect to the metric $g_{\min }$ :

$$
\begin{aligned}
\left|\left\langle D_{y} F_{s}\left(u_{i}\right), v_{i}\right\rangle_{g_{\min }}\right| & \leq \frac{\alpha_{i} \sqrt{n} \sqrt{n_{i}} s\left\|v_{i}\right\|_{g_{\min }}}{h_{i}}\left(\int_{\partial_{F} X} d B_{y, \phi^{-1}(\theta)}\left(u_{i}\right)^{2} d \sigma_{y}^{s}(\theta)\right)^{1 / 2} \\
& =\sqrt{n}\left\|v_{i}\right\|_{g_{\min }} \frac{s}{h\left(g_{\min }\right)}\left(\int_{\partial_{F} X} d B_{y, \phi^{-1}(\theta)}\left(u_{i}\right)^{2} d \sigma_{y}^{s}(\theta)\right)^{1 / 2}
\end{aligned}
$$

Hence for all $u \in T_{y} Y$ we obtain

$$
\left\|D_{y} F_{s}(u)\right\|_{g_{\min }} \leq \sqrt{n} \frac{s}{h\left(g_{\min }\right)}\left(\int_{\partial_{F} X} d B_{y, \phi^{-1}(\theta)}(u)^{2} d \sigma_{y}^{s}(\theta)\right)^{1 / 2}
$$

Now we follow Section 5 of [BCG2]. It follows that for any orthonormal basis $\left\{v_{i}\right\}$ of $T_{F_{s}(y)} X$ we have

$$
\operatorname{Tr}\left(D_{y} F_{s}^{*}\right) \circ\left(D_{y} F_{s}\right)=\sum_{i=1}^{n}\left\langle D_{y} F_{s}\left(v_{i}\right), D_{y} F_{s}\left(v_{i}\right)\right\rangle_{g_{\min }} \leq n\left(\frac{s}{h\left(g_{\min }\right)}\right)^{2}
$$

Lastly it follows that

$$
\begin{aligned}
\left(\frac{s}{h\left(g_{\min }\right)}\right)^{2}=\left|\operatorname{Jac} F_{s}\right|^{2} & =\operatorname{det}\left(D_{y} F_{s}\right)^{*} \circ\left(D_{y} F_{s}\right) \\
& \leq\left(\frac{\operatorname{Tr}\left(D_{y} F_{s}\right)^{*} \circ\left(D_{y} F_{s}\right)}{n}\right) \leq\left(\frac{s}{h\left(g_{\min }\right)}\right)^{2}
\end{aligned}
$$

The equality implies that

$$
\left(D_{y} F_{s}\right)^{*} \circ\left(D_{y} F_{s}\right)=\left(\frac{s}{h\left(g_{\min }\right)}\right)^{2} \mathrm{I}
$$

Hence $D_{y} F_{s}$ is a homothety of ratio $\frac{s}{h\left(g_{\min }\right)}$ as desired. 
Remark 1. The equality case in item (3) will not be used in what follows, but will provide insight into what happens as we take limits $s \rightarrow h(g)$.

\section{Finishing the proof of Theorem A.}

We now prove the statement given in Remark 1 after Theorem A, from which the theorem immediately follows. We first establish that $F_{s}$ is homotopic to $f:$

Proposition 6.1. For any $s>h(g)$, the map $\Psi_{s}:[0,1] \times N \rightarrow M$ defined by

$$
\Psi_{s}(t, y)=F_{s+\frac{t}{1-t}}(y)
$$

is a homotopy between $\Psi_{s}(0, \cdot)=F_{s}$ and $\Psi_{s}(1, \cdot)=f$.

Proof. From its definitions, $\tilde{F}_{s}(y)$ is continuous in $s$ and $y$. Observe that for fixed $y, \lim _{s \rightarrow \infty} \sigma_{y}^{s}=\nu_{\phi(y)}$. It follows that $\lim _{s \rightarrow \infty} \tilde{F}_{s}(y)=\phi(y)$. This implies the proposition.

\subsection{The compact case.}

Suppose $M$ and $N$ are compact. Since for $s>h(g), F_{s}$ is a $C^{1}$ map, we may simply compute the following using elementary integration theory:

$$
\begin{aligned}
& |\operatorname{deg}(f)| \operatorname{vol}(M)=|\operatorname{deg}(f)| \int_{M} d g_{\min }=\left|\int_{N} f^{*} d g_{\min }\right| \\
& \leq \int_{N}\left|F_{s}^{*} d g_{\min }\right| \quad=\int_{N}\left|\operatorname{Jac} F_{s} d g\right| \\
& =\int_{N}\left|\operatorname{Jac} F_{s}\right| d g \leq\left(\frac{s}{h\left(g_{\min }\right)}\right)^{n} \operatorname{vol}(N)
\end{aligned}
$$

Letting $s \rightarrow h(g)$ gives the inequality in Theorem A. In the case when

equality is achieved, after scaling the metric $g$ by the constant $\frac{h(g)}{h\left(g_{\min }\right)}$, we have $h(g)=h\left(g_{\min }\right)$ and $\operatorname{Vol}(N)=|\operatorname{deg}(f)| \operatorname{Vol}(M)$.

We remark that in the notation used in [BCG1] for the sequence of Lemmas 7.2-7.9 and Proposition 8.2, the measure $\Phi^{2}(y, \theta) d \theta$ is simply $\sigma_{y}^{s}$ in the rank one case (recall the Poisson kernel is $\left.p_{0}(y, \theta)=e^{-h(g) B_{0}(y, \theta)}\right)$.

In higher rank this equivalence still holds so long as we replace $d \theta$ by the Patterson-Sullivan measure $\nu_{p}$ at the basepoint $p$. The proofs of Lemmas 
7.2-7.9 and Proposition 8.2 in [BCG1] then hold in our more general context once we replace the visual boundary by the Furstenberg boundary and $d \theta$ by the measure $\nu_{p}$ as the analysis is the same. These show that $F_{s}$ converges to a nonexpanding Lipschitz map $F$ as $s \rightarrow h(g)$. Applying Theorem C.1 from Appendix C of [BCG1] we obtain Theorem A.

\subsection{The noncompact case.}

We now consider the case when $M$ has finite volume but is not compact. In this setting, it is not known whether the limit in the definition of $h(g)$ given for the compact case always exists. For analytic reasons we define the quantity $h(g)$ to be

$$
h(g):=\inf \left\{s \geq 0 \mid \exists C>0 \text { such that } \forall x \in X, \int_{X} e^{-s d(x, z)} d g(z)<C\right\} .
$$

In fact this agrees with the previous definition for $h(g)$ when $M$ is compact or $g$ is symmetric. Volume comparison implies $(n-1) a \leq h(g) \leq(n-1) b$ whenever $-a^{2}$ and $-b^{2}$ are upper and lower bounds for the sectional curvatures. Moreover, $h(g)$ is conjecturally the same as $\lim \sup _{R \rightarrow \infty} R^{-1} \log \operatorname{vol} B(x, R)$ for any $x \in X$.

If $N$ has infinite volume the main theorem is automatically satisfied so long as $h(g)>0$. The main difficulty is that, in order for the proof given above to work, we need to know that $F_{s}$ is proper (and thus surjective since $\left.\operatorname{deg}\left(F_{s}\right)=\operatorname{deg}(f) \neq 0\right)$. For this, we will need to prove higher rank analogs of some lemmas used in [BCS] for the rank one case. For the basics of degree theory for proper maps between noncompact spaces, see [FG].

We will show that $F_{s}$ is proper by essentially showing that the barycenter of $\sigma_{x}^{s}$ lies nearby a convex set containing large mass for this measure. This convex set is in turn far away from $\phi(p)$ whenever $x$ is far from $p \in Y$. We achieve this by first estimating the concentration of the mass of $\sigma_{x}$ in certain cones which will be our convex sets. One difficulty that arises in the higher rank is that these cones must have a certain angle when restricted to a flat. Another difficulty is that the ends of $M$ can have large angle at infinity. In fact our methods breakdown unless we control the asymptotic expansion of $f$ down the ends (see Remarks 2).

First, we localize the barycenter of the measure $\sigma_{x}^{s}$. Let $v_{(x, \theta)}$ be the unit vector in $S_{x} X$ pointing to $\theta \in \partial X$.

Lemma 6.2. Let $K \subset X$ and $y \in Y$ be such that $\left(\phi_{*} \mu_{y}^{s}\right)(K)>C$ for some constant $1>C>\frac{1}{2}$. Suppose that for all $x \in X$ there exists $v \in S_{x} X$ such 
that for all $z \in K$ :

$$
\int_{\partial_{F} X}\left\langle v_{(x, \theta)}, v\right\rangle d \nu_{z}(\theta) \geqslant \frac{1}{C}-1
$$

Then

$$
x \neq \tilde{F}_{s}(y)
$$

Proof. If $\tilde{F}_{s}(y)=x$ then $\nabla_{x} \mathcal{B}_{s, y}(x)=0$. However, $\nabla_{x} \mathcal{B}_{s, y}(x)$ may be expressed as

$$
\int_{X} \int_{\partial_{F} X} v_{(x, \theta)} d \nu_{z}(\theta) d \phi_{*} \mu_{y}^{s}(z)
$$

where $v_{(x, \theta)}$ is the unit vector in $S_{x} X$ pointing to $\theta \in \partial_{F} X$. Then we have

$$
\begin{aligned}
\left\|D_{x} \mathcal{B}_{s, y}\right\| & =\left\|\int_{X} \int_{\partial_{F} X} v_{(x, \theta)} d \nu_{z}(\theta) d \phi_{*} \mu_{y}^{s}(z)\right\| \\
& \geqslant\left\|\int_{K} \int_{\partial_{F} X} v_{(x, \theta)} d \nu_{z}(\theta) d \phi_{*} \mu_{y}^{s}(z)\right\|- \\
& \geqslant \int_{K} \int_{\partial_{F} X}\left\langle\int_{X-K} \int_{\partial_{F} X} v_{(x, \theta)} d \nu_{z}(\theta) d \phi_{*} \mu_{y}^{s}(z) \| d \nu_{z}(\theta) d \phi_{*} \mu_{y}^{s}(z)-\phi_{*} \mu_{y}^{s}(X-K)\right. \\
& \geqslant \phi_{*} \mu_{y}^{s}(K)\left(\frac{1}{C}-1\right)-1+\phi_{*} \mu_{y}^{s}(K) \\
& >C\left(\frac{1}{C}-1\right)-1+C=0
\end{aligned}
$$

The strictness of the inequality finishes the proof.

For $v \in S X$ and $\alpha>0$ consider the convex cone,

$$
E_{(v, \alpha)}=\exp _{\pi(v)}\left\{w \in T_{\pi(v)} X \mid \angle_{\pi(v)}(v(\infty), w(\infty)) \leq \alpha\right\}
$$

where $\pi: T X \rightarrow X$ is the tangent bundle projection.

Denote by $\partial E_{(v, \alpha)} \subset \partial X$ its boundary at infinity. 
Lemma 6.3. There exists $T_{0}>0$ and $\alpha_{0}>0$ such that for all $t \geq T_{0}$, all $x \in X$, all $v \in S_{x} X$ and all $z \in E_{\left(g^{t} v, \alpha_{0}\right)}$,

$$
\int_{\partial_{F} X}\left\langle v_{(x, \theta)}, v\right\rangle d \nu_{z}(\theta) \geqslant \frac{\sqrt{2}}{3} .
$$

Proof. Since the isometry group of the symmetric space $X$ is transitive on $X$ and for any isometry $\psi, d \psi\left(E_{(v, \alpha)}\right)=E_{(d \psi(v), \alpha)}$, it is sufficient to prove the lemma for a fixed $x$ and all $v \in S_{x} X$.

For now choose $\alpha_{0}<\pi / 4$. Take a monotone sequence $t_{i} \rightarrow \infty$, and any choice $z_{i} \in E_{\left(g^{\left.t_{i}, \alpha\right)}\right)}$ for each $t_{i}$. It follows that some subsequence of the $z_{i}$, which we again denote by $\left\{z_{i}\right\}$, must tend to some point $\theta \in \partial E_{(v, \alpha)}$.

Let $\nu_{\theta}$ be the weak limit of the measures $\nu_{z_{i}}$. From Theorem 3.3, $\nu_{\theta}$ is a probability measure supported on a set $S_{\theta}$ satisfying

$$
\angle_{x}(\theta, \xi) \leq \frac{\pi}{4} \quad \forall \xi \in S_{\theta}
$$

Therefore we have,

$$
\int_{S_{\theta}}\left\langle v_{(x, \xi)}, v_{(x, \theta)}\right\rangle d \nu_{\theta}(\xi) \geqslant \frac{\sqrt{2}}{2}
$$

Now whenever $\theta \in \partial E_{(v, \alpha)}$ then $v=v_{(x, \theta)}+\epsilon v^{\prime}$ for some unit vector $v^{\prime}$ and $\epsilon \leq \sin (\alpha)$. Using either case above we may write

$$
\int_{\partial_{F} X}\left\langle v_{(x, \xi)}, v\right\rangle d \nu_{\theta}(\xi) \geqslant \int_{\partial_{F} X}\left\langle v_{(x, \xi)}, v_{(x, \theta)}\right\rangle d \nu_{\theta}(\xi)-\sin (\alpha) .
$$

So choosing $\alpha$ small enough we can guarantee that

1. any two Weyl chambers intersecting $E_{\left(g^{t} v, \alpha\right)}$ for all $t>0$ in the same flat must share a common face of dimension $\operatorname{rank}(M)-1$, and

2. for any $\theta \in \partial E_{(v, \alpha)}$,

$$
\int_{\partial_{F} X}\left\langle v_{(x, \xi)}, v\right\rangle d \nu_{\theta}(\xi) \geqslant \frac{\sqrt{2}}{2.5} .
$$

Let

$$
E_{(v(\infty), \alpha)}=\cap_{t>0} \partial E_{\left(g^{t} v, \alpha\right)}
$$


By the first property used in the choice of $\alpha$ above, for any two points $\theta_{1}, \theta_{2} \in E_{(v(\infty), \alpha)}$, either $\theta_{1}$ and $\theta_{2}$ are in the boundary of the same Weyl chamber, or else there is another point $\theta^{\prime}$ in the intersection of the boundaries at infinity of the closures of the respective Weyl chambers.

By maximality there is some $\theta_{0} \in E_{(v(\infty), \alpha)}$ intersecting the boundary at infinity of the closure of every Weyl chamber which intersects $E_{\left(g^{t} v, \alpha\right)}$ for all $t>0$. Hence, for every $\theta \in E_{(v(\infty), \alpha)}$, the support of the limit measure $\nu_{\theta}$ satisfies $S_{\theta} \subset S_{\theta_{0}}$. (While $\theta_{0}$ is not necessarily unique, the support $S_{\theta_{0}}$ of the corresponding limit measure $\nu_{\theta_{0}}$ is.)

As $t$ increases, for any $z \in E_{\left(g^{t} v, \alpha\right)}$, the measures $\nu_{z}$ uniformly become increasingly concentrated on $S_{\theta_{0}}$. Then applying the estimate (6) to $\theta=\theta_{0}$, we may choose $T_{0}$ sufficiently large so that for all $z \in E_{\left(g^{t} v, \alpha\right)}$ with $t>T_{0}$,

$$
\int_{\partial_{F} X}\left\langle v_{(x, \xi)}, v\right\rangle d \nu_{z}(\xi) \geqslant \frac{\sqrt{2}}{3} .
$$

Proposition 6.4. $F_{s}$ is proper.

Proof. By way of contradiction, let $y_{i} \in Y$ be an unbounded sequence such that $\left\{\tilde{F}_{s}\left(y_{i}\right)\right\}$ lies in a compact set $K$. We may pass to an unbounded subsequence of $\left\{y_{i}\right\}$, which we again denote as $\left\{y_{i}\right\}$, such that the sequence $\phi\left(y_{i}\right)$ converges within a fundamental domain for $\pi_{1}(M)$ in $X$ to a point $\theta_{0} \in \partial X$. Let $T_{0}$ and $\alpha_{0}$ be given from Lemma 6.3. Since $K$ is compact, the set

$$
A=\bigcap_{x \in K} E_{\left(g^{T_{0}} v_{\left(x, \theta_{0}\right)}, \alpha_{0}\right)}
$$

contains an open neighborhood of $\theta_{0}$ and $d_{X}(A, K) \geq T_{0}$. Notice that $A$ is itself a cone, being the intersection of cones on a nonempty subset of $\partial X$.

We now show that $A$ contains the image $\phi\left(B\left(y_{i}, R_{i}\right)\right)$ of increasingly large balls $\left(R_{i} \rightarrow \infty\right)$, and eventually use this to derive a contradiction. We observe from the fact that $A$ is a cone on an open neighborhood of $\theta_{0}$ in $\partial X$ that $A$ contains balls $B\left(\phi\left(y_{i}\right), r_{i}\right)$ with $r_{i} \rightarrow \infty$. By assumption $f$, and hence $\phi$, is coarsely Lipschitz:

$$
d_{X}(\phi x, \phi y) \leq K d_{Y}(x, y)+C
$$

for some constants $C>0$ and $K \geq 1$. Therefore $\phi^{-1}\left(B\left(\phi\left(y_{i}\right), r_{i}\right)\right) \supset$ $B\left(y_{i}, R_{i}\right)$ where $K R_{i}+C>r_{i}$. In particular $R_{i} \rightarrow \infty$. 
Hence, there exists an unbounded sequence $R_{i}$ such that $B\left(y_{i}, R_{i}\right) \subset$ $\phi^{-1}(A)$. Furthermore, since $Y$ has bounded geometry, we have that $\operatorname{vol}\left(B\left(y_{i}\right.\right.$, injrad $\left.)\right)$ is greater than some constant independent of $y_{i}$ and hence

$$
\int_{Y} e^{-s d\left(y_{i}, z\right)} d g(z)>Q
$$

for some constant $Q>0$. By choice of $s$ there is a constant $C_{s}$ depending only on $s$ such that $\int_{Y} e^{-s d(y, z)} d g(z)<C_{s}$ for all $y \in Y$.

In polar coordinates we may write,

$$
\begin{aligned}
\int_{Y} e^{-s d(y, z)} d g(z) & =\int_{0}^{\infty} e^{-s t} \operatorname{vol}(S(y, t)) d t \\
& =\int_{0}^{\infty} e^{-s t} \frac{d}{d t} \operatorname{vol}(B(y, t)) d t \\
& =-\int_{0}^{\infty} \frac{d}{d t}\left(e^{-s t}\right) \operatorname{vol}(B(y, t)) d t \\
& =s \int_{0}^{\infty} e^{-s t} \operatorname{vol}(B(y, t)) d t
\end{aligned}
$$

Using this we may estimate, using any $\delta<s-h(g)$,

$$
\begin{aligned}
\mu_{y_{i}}^{s}\left(\phi^{-1}(A)\right) & >\mu_{y_{i}}^{s}\left(B\left(y_{i}, R_{i}\right)\right) \\
& =1-\frac{\int_{R_{i}}^{\infty} e^{-s t} \operatorname{vol}\left(B\left(y_{i}, t\right)\right) d t}{\int_{0}^{\infty} e^{-s t} \operatorname{vol}\left(B\left(y_{i}, t\right)\right) d t} \\
& \geq 1-\frac{e^{-\delta R_{i}} \int_{R_{i}}^{\infty} e^{-(s-\delta) t} \operatorname{vol}\left(B\left(y_{i}, t\right)\right) d t}{\int_{0}^{\infty} e^{-s t} \operatorname{vol}\left(B\left(y_{i}, t\right)\right) d t} \\
& \geq 1-e^{-\delta R_{i}} \frac{C_{s-\delta}}{Q} .
\end{aligned}
$$

Therefore for all sufficiently large $i$,

$$
\mu_{y_{i}}^{s}\left(\phi^{-1}(A)\right)>\frac{3}{3+\sqrt{2}}
$$

The constant $\frac{3}{3+\sqrt{2}}$ is the constant $C$ from Lemma 6.2 such that $\frac{1}{C}-1=\frac{\sqrt{2}}{3}$.

Set $v_{i}=g^{T_{0}+1} v_{\left(\tilde{F}_{s}\left(y_{i}\right), \theta_{0}\right)}$. Recalling that $A \subset E_{\left(v_{i}, \alpha_{0}\right)}$ for all $i$, we have that for sufficiently large $i$,

$$
\phi_{*} \mu_{y_{i}}^{s}\left(E_{\left(v_{i}, \alpha_{0}\right)}\right)>\frac{3}{3+\sqrt{2}}
$$


but $d_{X}\left(\tilde{F}_{s}\left(y_{i}\right), E_{\left(v_{i}, \alpha_{0}\right)}\right)>T_{0}$, contradicting the conclusion of Lemma 6.2 in light of Lemma 6.3.

Remark 2. It is likely that ideas from coarse topology can be used to remove the coarse lipschitz assumption on $f$ in the case that the ends of $M$ have angle at infinity bounded away from $\pi / 2$. Nevertheless, $M$ may have ends containing pieces of flats with wide angle (consider the product of two rank one manifolds each with multiple cusps, or refer to the classification of higher rank ends in $[\mathrm{Ha}])$. For such spaces it is possible to construct a proper map $f: M \rightarrow M$ such that for a radial sequence $y_{i} \rightarrow \infty, \phi$ maps the bulk of the mass of $\mu_{y_{i}}^{s}$ into a set (nearly) symmetrically arranged about the point $p \in X$ thus keeping $\tilde{F}_{s}\left(y_{i}\right)$ bounded. This explains the need for a condition on $f$ akin to the coarse lipschitz hypothesis.

The inequality in Theorem A now follows as in the compact case, with $\operatorname{deg}(f)$ and $\operatorname{deg}\left(F_{s}\right)$ suitably interpreted.

Now we complete the proof of the rigidity when we have equality in Theorem A. First we assume that $h(g)=h\left(g_{0}\right)$ by scaling the metric $g$ by the constant $\frac{h\left(g_{0}\right)}{h(g)}$. We note that the proofs of the lemmas in Section 7 of [BCG1] (done for the case $f=\mathrm{Id}$ ) are identical so long as we restrict the uniformity of Lemmas 7.5 and 7.6 to be uniform only on compact subsets. These proofs go through with only minor modification in the case that $f$ has (local) degree $\operatorname{deg}(f) \neq 1$; this is explained in Section 8.2 of [BCG1]. In this case we obtain the general version of Lemma 7.6 of [BCG1],

Lemma 6.5. There is a subsequence $s_{i}$ such that the maps $F_{s_{i}}$ converges uniformly on compact sets to a continuous map $F: N \rightarrow M$.

The proofs of Lemma 7.7 and 7.8 of [BCG1] then go through without modification to obtain,

Lemma 6.6. The sequence $\left\|d_{y} F_{s_{i}}\right\|$ is uniformly bounded for $y$ in compact subsets of $M$ and converges to 1 almost everywhere. The map $F$ is Lipschitz with Lipschitz constant less than or equal to one.

It does not follow from the proof of Proposition 6.4 that the $F_{s}$ are uniformly proper since the radii $R$ appearing there become unbounded as $s \rightarrow h(g)$. Nevertheless, we can achieve this with a little more work.

Lemma 6.7. The image of $F$ has full Lebesgue measure and $F^{-1}(y)$ is a finite set for almost every $y \in M$. 
Proof. Let $K_{1} \subset K_{2} \subset \cdots \subset K_{i} \subset \ldots$ be a sequence of relatively compact open sets exhausting $N$. Setting $U_{i}=N \backslash K_{i}$, for each $\epsilon>0$ there is an $i(\epsilon) \in \mathbb{N}$ such that $\operatorname{vol}\left(U_{i}\right)<\epsilon$ for all $i>i(\epsilon)$.

Since the $F_{s_{i}}$ are surjective and uniformly converge to $F$ on compacta, for each $i$ there is a $j(i) \in \mathbb{N}$ such that $(M \backslash F(N)) \subset F_{s_{j}}\left(U_{i}\right)$ for all $j>j(i)$. However by Theorem 5.1 , we have $\left|\operatorname{Jac}\left(F_{s}\right)\right| \leq\left(\frac{s}{h\left(g_{\min }\right)}\right)^{n}$. Therefore,

$$
\left(\frac{s}{h\left(g_{\text {min }}\right)}\right)^{n} \operatorname{vol}\left(U_{i}\right) \geq \int_{U_{i}}\left|\operatorname{Jac}\left(F_{s}\right)\right| d g \geq \int_{F_{s}\left(U_{i}\right)} N_{f} d g_{\text {min }},
$$

where for $y \in M, N_{f}(y)=\operatorname{card}\left\{x \in f^{-1}(y) \cap U_{i}\right\}$. Since $N_{f}(y) \geq 1$ for $y \in F_{s}\left(U_{i}\right)$, the right hand side is larger than $\operatorname{vol}\left(F_{s}\left(U_{i}\right)\right)$. So we have $\operatorname{vol}\left(F_{s}\left(U_{i}\right)\right) \leq\left(\frac{s}{n-1}\right)^{n} \operatorname{vol}\left(U_{i}\right)$ and therefore the volume of $F_{s_{j}}\left(U_{i}\right)$ tends to 0 as $i \rightarrow \infty$ independently of $j$. This gives the first statement.

The second statement follows from the observation that the set $C$ of point $y$ such that $F^{-1}(y)$ is infinite is contained in $F(N)^{\complement} \cup \bigcap_{i=1}^{\infty} F\left(U_{i}\right)$ which have just shown has measure 0 .

For any Lebesgue mesureable subset $A \subset N$ and any Lipschitz function $f: A \rightarrow M$ and for almost every $y \in M$ the function degree $\left.f\right|_{A}$, given by

$$
\operatorname{degree}\left(\left.f\right|_{A}\right)(y):=\sum_{x \in f^{-1}(y) \cap A} \operatorname{sgn}[(\operatorname{Jac} f)(x)]
$$

exists in $\mathcal{L}^{1}(M)$. We write degree $(f)$ for degree $\left(\left.f\right|_{N}\right)$.

Lemma 6.8. For any measurable set $A \subset N$ with $\operatorname{vol}(\partial A)=0$ and almost every $y \in M$ we have $\operatorname{degree}\left(\left.F\right|_{A}\right)(y)=\lim _{j \rightarrow \infty} \operatorname{degree}\left(\left.F_{s_{j}}\right|_{A}\right)(y)$, and in particular degree $(F)(y)=\operatorname{deg}(f)$.

Proof. For any $s>n-1, F_{s}$ is $C^{1}$ and proper homotopic to $f$ and hence $\operatorname{degree}\left(F_{s}\right)(y)=\operatorname{deg}(f)$ for every $y \in M$, so it is enough to show the first formula.

By the previous lemma we may assume $F^{-1}(y)$ is finite and therefore that $A$ is relatively compact. Since $F_{s_{j}}$ converges uniformly to $F$ on compacts, we have for $x \in A, d\left(F_{s_{j}}(x), F(x)\right)<\epsilon(j)$ where $\epsilon(j) \rightarrow 0$ as $j \rightarrow \infty$.

For some open subsets $U, U_{o} \subset \mathbb{R}^{n}$, let $\phi: U \rightarrow N$ and $\phi_{o}: U_{o} \rightarrow M$ be diffeomorphisms onto their respective images such that their derivatives have norm bounded above and below. Without loss of generality, we may assume 
That $\phi(U)$ and $\phi_{o}\left(U_{o}\right)$ have full measure. Lastly, let $W \subset \phi^{-1}(A) \cap U$ be any subset with $\operatorname{vol}(\partial W)=0$ and let $h:[0,1] \times \mathbb{R}^{n} \rightarrow \mathbb{R}^{n}$ be the homotopy $h(t, x)=(1-t) \phi_{o}^{-1} F_{s_{j}}(\phi x)+t \phi_{o}^{-1} F(\phi x)$.

Recall that the norms of the derivatives of $F_{s_{j}}$ and $F$ for sufficiently large $j$ are both almost everywhere bounded by 2 . It follows that the norms of the derivatives of $h$ are bounded above by a constant $C$ almost everywhere. Define the current $T:=\left(\operatorname{vol}_{\mathbb{R}^{n}} \bigwedge_{i=1}^{n} e_{i}\right)\left\llcorner W\right.$ where $\bigwedge_{i=1}^{n} e_{i}$ is the standard $n$ frame field in $\mathbb{R}^{n}$. The estimate 4.1.13 in [Fe] remains valid in the Lipschitz setting as shown at the end of 4.1.14. Applied to the current $T$ and combined with Corollary 4.1.26 in [Fe], this shows

$$
\begin{gathered}
\int_{h([0,1] \times W)} \operatorname{degree}\left(\left.F\right|_{A}\right)(y)-\operatorname{degree}\left(\left.F_{s_{j}}\right|_{A}\right)(y) d g_{\min }< \\
C^{m+1}\left(\int_{\bar{W}}\left|\phi_{o}^{-1} F_{s_{j}}(\phi x)-\phi_{o}^{-1} F(\phi x)\right| d g\right) .
\end{gathered}
$$

(Here we have also used that spt $\partial T=\partial W$ is a null set along with the definitions in 4.1.7 and 4.1.12 of [Fe], and that for any Lipschitz map $F$ and diffeomorphisms $\phi$ and $\phi_{o}$, degree $\left(\left.F\right|_{A}\right)(y)=\operatorname{degree}\left(\left.\phi_{o}^{-1} \circ F \circ \phi\right|_{\phi^{-1}(A)}\right)\left(\phi_{o}^{-1} y\right)$.)

Taking limits as $j \rightarrow \infty$ we obtain $\int_{F(W)} G(y) d g_{\min }=0$ where $G(y)=$ degree $\left(\left.F\right|_{A}\right)(y)-\lim _{j \rightarrow \infty} \operatorname{degree}\left(\left.F_{s_{j}}\right|_{A}\right)(y)$. The lemma follows from the fact that we may arbitrarily approximate in $\mathcal{L}^{1}$ the supports of the functions $G_{+}=\{y \mid G(y) \leq 0\}$ and $G_{-}=\{y \mid G(y)<0\}$ by sets $F(W)$ with $\operatorname{vol}(\partial W)=$ $\operatorname{vol}(F(\partial W))=0$ since $F$ is Lipschitz.

Lemma 6.9. For any Lebesgue measurable set $A \subset M$, we have $\operatorname{vol}\left(F^{-1}(A)\right)=\operatorname{deg}(f) \operatorname{vol}(A)$.

Proof. Since $F$ is 1-Lipschitz, $-1 \leq \operatorname{Jac}(F) \leq 1$ almost everywhere. Lemma 4.1.25 and Corollary 4.1.26 of [Fe] show

$$
\operatorname{vol}\left(F^{-1} A\right) \geq \int_{F^{-1}(A)} \operatorname{Jac}(F) d g=\int_{A} \text { degree }\left.F\right|_{F^{-1}(A)} d g_{\min } .
$$

However, by the previous Lemma,

$$
\int_{A} \operatorname{degree}\left(\left.F\right|_{F^{-1} A}\right) d g_{\min }=\int_{A} \operatorname{degree}(F) d g_{\min }=\operatorname{deg}(f) \operatorname{vol}(A) .
$$

On the other hand, Lemma 6.7 implies $\operatorname{vol}(N)=\operatorname{vol}\left(F^{-1} A\right)+\operatorname{vol}\left(F^{-1} A^{\complement}\right) \geq \operatorname{deg}(f)\left(\operatorname{vol}(A)+\operatorname{vol}\left(A^{\complement}\right)\right)=\operatorname{deg}(f) \operatorname{vol}(M)$. 
The fact that we have equality here finishes the lemma.

The next Proposition finishes the proof of the Main Theorem. We indicate how this is essentially proven in Appendix C of [BCG1].

Proposition 6.10. The map $F$ is a (proper) Riemannian cover of degree $\operatorname{deg}(f)$.

Proof. The proofs of Lemmas C.2 and C.4 in [BCG1] continue to apply once we know Lemmas 6.7 and 6.9. From these we obtain that for every $x \in U$, $\operatorname{card}\left(F^{-1}(x)\right) \leq \operatorname{deg}(f)$.

In particular, the preimage set $\left\{F^{-1}(x)\right\}$ is compact and $F$ is proper. The rest of the proofs of Appendix $\mathrm{C}$ can then be followed verbatim to show that for every $x \in N, \operatorname{card}\left(F^{-1}(x)\right)=\operatorname{deg}(f)$ and $F$ is a local isometry onto $U$.

\section{References.}

[Al] P. Albequerque, Patterson-Sullivan theory in higher rank symmetric spaces, Geom. Funct. Anal. (GAFA), Vol. 9 (1999), 1-28.

[BCG1] G. Besson, G. Courtois and S. Gallot, Entropies et rigidités des espaces localement symétriques de courbure strictement négative, . Geom. Funct. Anal. 5 (1995), no. 5, 731-799.

[BCG2] Minimal entropy and Mostow's rigidity theorems, Ergodic Theory Dynam. Systems 16 (1996), no. 4, 623-649.

[BCG3] , Volumes and rigidities of Riemannian manifolds, January 1999, in preparation.

[BCS] J. Boland, C. Connell, and J. Souto, Minimal entropy rigidity for finite volume manifolds, Preprint, 1999.

[BGS] W. Ballmann, M. Gromov and V. Schroeder, Manifolds of Nonpositive Curvature, Progress in Mathematics, Vol. 61, Birkaüser, 1985.

[Eb] P. Eberlein, Geometry of nonpositively curved manifolds, Chicago Lectures in Math. Univ. of Chicago Press, 1996.

[Fe] Federen H.: Geometric measure theory. Die Grundlehren der mathematischen Wissenschaften, Band 153. New York, 1969. 
[FG] I. Fonseca and W. Gangbo, Degree theory in analysis and applications, The Clarendon Press Oxford University Press, New York, 1995, Oxford Science Publications.

[GJT] Y. Guivarc'h, L. Ji, and J. C. Taylor, Compactifications of symmetric spaces, Progress in Mathematics, Vol. 156, Birkaüser, 1998.

[Ha] T. Hattori, Asymptotic geometry of arithmetic quotients of symetric spaces, Math. Z. 222 (1996), no. 2, 247-277.

[Kn] G. Knieper, On the asymptotic geometry of nonpositively curved manifolds, Geom. Funct. Anal. 7 (1997), no. 4, 755-782.

[Ma] A. Manning, Topological entropy for geodesic flows, Annals of Math. 110 (1979), 567-573.

[Ve] P. Verovic, Probleme de l'entropie minimale pour les métriques de Finsler, Erg. Theo. and Dynam. Syst., 19 (1999), no. 6, 1637-1654.

[Zi] R. Zimmer, Ergodic Theory and Semisimple Groups, Monographs in Math., Vol. 81, Birkhäuser, 1984.

\section{Christopher Connell}

DePt. of Mathematics, University of Illinois at Chicago

Chicago, IL 60680

cconnell@math.uic.edu

\section{BENSON FARB}

Dept. of Mathematics, University of Chicago

5734 University Ave.

ChICAGo, IL 60637

farb@math.uchicago.edu

Received February 7, 2002. 